\title{
Ultrastructural pathology of nasal and tracheal mucosa of rabbits experimentally infected with Pasteurella multocida serotype D:1
}

\begin{abstract}
Sixteen 8- to 9-week-old Pasteurella multocida-free New Zealand White rabbits were divided into two equal groups. The first group was inoculated intranasally with $\mathrm{P}$ multocida serotype D:1 strain and the second group that was inoculated with phosphate-buffered saline (PBS) only was used as a control group. Pasteurella multocida was isolated from the nasal cavity of all infected rabbits in group 1 and from tracheal swabs of seven rabbits in this group. Four rabbits in group 1 died with clinical signs of septicaemia, two rabbits had mucopurulent nasal discharge and pneumonic lesions and the other two did not show any clinical signs or gross lesions. The ultrastructural changes detected were deciliation or clumping of cilia of ciliated epithelium, cellular swelling, vacuolation and sloughing. The subepithelial capillaries showed congestion, intravascular fibrin deposition, platelets aggregation and endothelial injury. Pasteurella multocida was observed attached to the injured endothelial cells. Heterophils, mast cells, vacuolated monocytes and macrophages infiltrated the lamina propria and between the degenerated epithelial cells. (C2001 Harcourt Publishers Ltd
\end{abstract}

